\title{
MOBILITY MANAGEMENT AND PERFORMANCE STATISTICS IN CELLULAR COMMUNICATION
}

\author{
${ }^{1} \mathrm{C}$ Ufuah, ${ }^{2} \mathrm{~K} O$ Tanno
}

\author{
Delta State Polytechnic Otefe-Oghara, Nigeria
}

\begin{abstract}
This study presents and examines a management model of cellular mobility protocols and the performance of some of its parameters for optimal spectral efficiency of the mobile communication system. Mobility management enables the networks to track the location of mobile nodes. Location management encompasses location registration and call delivery or paging. With the convergence of the internet and wireless mobile communications and with the rapid growth in the number of mobile subscribers, mobility management emerges as one of the most important and challenging problems for wireless mobile communication over the internet. This work is presented on the platform of an analytical framework that can enhance considerably the mobility mechanism in wireless network. Some advance schemes namely, guard channels, and handover queuing are discussed. All these prioritizations schemes have a common characteristic reducing the call dropping probability at the expense of increased call blocking probability. The mobility management scheme addressed latency in the service delivery enhancing the guaranteed quality of service (Qos) and an efficient and robust channel capacity.
\end{abstract}

\section{INTRODUCTION}

Mobility management is extremely important in cellular network because of the cellular architecture employed to maximize spectrum utilization. Mobility is the procedure that transfers an ongoing call from one cell to another as the user's moves through the coverage area of cellular system. One way to improve the cellular network performance is to use efficient handover prioritization schemes when user is switching between the cells. Efficient prioritization scheme accommodates a number of new calls while guarantees the quality of service (QoS) of handover call.Over the past years, a number of IP mobility management protocols have been proposed. Different mobility management frameworks can be broadly distinguished into two categories - device mobility management protocol localized or micro-mobility and protocol for inter-domains or macro mobility. The movement of a mobile node (MN) between two subnets within the domain is referred to as micro-mobility.

Mobility management enables the serving networks to locate a mobile subscriber's point or attachment for delivering data packets (ie location management), and maintain a mobile subscriber's connection as it to change its point of attachment.

Domains: A domain represents an administrative body which may include different access network such as WLAN, second generation (2G) and third generation (3G) networks. Next - generation all - IP wireless network will induce various heterogenic networks, each of them using possibly different access technologies.

A handover is a process in telecommunications and mobile communications in which a connected cellular call or data session is transferred from one cell site (base station) to another without disconnecting the session. Cellular services are based on mobility and handover, allowing the user to be moved from one cell site range to another to be switched to the nearest cell site for better performance Ekici (2012).

The progressive trend of urbanization involving changes in the activities of a city has created several problems. Addressing these problems requires reliable and detailed information regarding the urban structure and its dynamics. In order to avoid abrupt termination of ongoing call handover request should be given priority to new call this is called as mobility prioritization.

There are two techniques for this.

Guard Channel Concept: In this technique, a fraction of the total available channel in a cell is reserved exclusively for handover request from ongoing calls which may be handed off into the cell.

2) Queuing: Queuing of handoffs is possible because there is a finite time interval between the time the received signal level drops below handoff threshold and the time the call is terminated due to insufficient signal level. The delay size is determined from the traffic pattern of a particular service area Misra, Das and McAuley (2010).

This is the process by which a mobile mode keeps its connection active when it moves from one access point to another. There are stages in the mobility process. First, the initiation of handover is triggered by either the mobile device or a network agent or the changing network conditions. The second stage is for a new connection generation when the network must find new resources for the handover connection and perform and additional routing operations. Depending on the movement of the mobile device, it may undergo various type of handover. In a broad sense, handover may be intra-system (horizontal handover) which occurs in homogenous networks. The type occurs when the signal strength or the serving BS goes below a certain threshold value. Handover 
on the other hand may also be inter-system (vertical handover) which takes place in heterogeneous networks when a user moves out or the serving network and enters and overlying network or to underlying network for service requirement.

In essence, the design or mobility management techniques in all wireless networks must address the following issues: (1) signaling overhead and power requirement for processing handover messages should be minimized. (ii) Qos guarantees must be made (iii) network resources should be efficiently used and (iv) the mobility mechanism should be scalable viable and robust.

With the convergence of the internet and wireless mobile communications and with the rapid growth in the number of mobile subscribers, mobility management emerges as one of the most important and challenging problems for wireless mobile communication over the internet. Mobility management enables the serving networks to locate a mobile subscriber's point or attachment for delivering data packets (ie location management) and maintain a mobile subscriber's connection as it to change its point of attachment.

Location management enables the networks to track the location of mobile nodes. Location management has two major sub-tasks (i) location registration on (ii) call delivery or paging. In location registration procedure, the mobilizers node periodically sends specific signals the inform the network of its current location so that the location database it kept update.

The call delivery proceeds in involved after the completion or the location registration. Based on the information that has been registered in the network during the location registration.

The overall aim of this study is to enhance the concept of cellular mobility protocols and investigate some critical parameters to validate the concept.

In order to achieve the aim of this study, the following objectives are set out as outlined below.

(a) Ascertain call set up time to enhance efficiency and effectiveness of calls made by a mobile station.

(b) Optimize the quality of service (QoS) as a critical performance indicator of the network for efficient service delivery.

\section{Technology Developments}

The radio spectrum is a precious and scarce resource. Therefore, novel technologies for efficient spectrum utilization to enhance the capacity of $3 \mathrm{G}$ and beyond systems are keenly anticipated. Factors that could have a significant impact on the deployment of mobile telecommunications technologies in this timeframe include radio access techniques enabling greater intelligence and flexibility to be built into transmitters and receivers. Some technology topics that appear relevant to some lesser or greater degree to the future development are: advanced radio resource management (RRM) algorithms; flexible frequency sharing methods; smart antennas; diversity techniques; coding techniques; space-time coding; efficient multiple access schemes or adaptive modulation.

$3 \mathrm{G}$ mobile communications systems arose as a response to the challenge of developing systems that increased the capacity of the existing $2 \mathrm{G}$ systems. Simultaneously, they would provide a platform that allowed a seamless and ubiquitous access to the user of a wide range of new services, both circuit and packet switched, with higher requirements in terms of bit rate than those for which $2 \mathrm{G}$ systems were conceived. The development of 3G systems started in 1995, coordinated by the ITU-T (International Telecommunications Union - Telecommunications sector) under the generic terminology of IMT-2000 and so far different radio access technologies have been considered leading to the development of several standards. Within this framework, the Universal Mobile Telecommunications System (UMTS) is the European proposal given by ETSI (European Telecommunications Standards Institute) to the $3 \mathrm{G}$ challenge. As a matter of fact, it is the dominant standard, resulting from the standardization work done by the 3GPP (3rd Generation Partnership Project), an organization formed by different regional standardization bodies that include the presence of both manufacturers and operators from all around the world.

UMTS has been developed as the migration of the ETSI $2 \mathrm{G} / 2.5 \mathrm{G}$ systems GSM/GPRS. The aim is to facilitate as much as possible the extension of the existing networks of these worldwide systems as well as the interoperability of the new UMTS system with the previous networks, thus allowing a progressive migration of the technology. As a result of this requirement, the most important changes introduced in the initial release of UMTS consist of a new radio access network based on a different radio access technology, while keeping the core network similar to that existing in GSM/GPRS systems.

The general UMTS network architecture from the physical point of view is presented here and it consists of an abstract model, applicable to any UMTS network, with independency of the specific release. It is organised in domains, and each domain represents the highest level group of physical entities. Reference points are defined between the different domains. The basic split considers the User Equipment (UE) domain, used by the user to access the UMTS services, and the Infrastructure domain, UMTS 7 composed of the physical nodes, belonging to the network operator, that support the service requirements and the interconnection with the entity at the other end (e.g. another UE from the same or another network) with whom the end-to-end service has to be established. 
The infrastructure domain in the UMTS architecture contains the physical nodes that terminate the radio interface allowing the provision of the end-to-end service to the UE. In order to separate the UMTS Subscriber Identity Module (USIM) domain.

UMTS 11 supporting one or both of the two modes, as well as the ability to interoperate between them. Notice that, from the radio resource management point of view, the concept of radio resource is different for each mode. As a result, the RRM strategies in both cases lead to different types of algorithms. In the context of this book, only the RRM strategies for the UTRAN FDD mode are considered.

UTRAN. Quality requirements expressed by, for example, delay and reliability attributes, only apply to incoming traffic up to the guaranteed bit rate. It is worth noting that the guaranteed bit rate at the RAB level may be different from that on the UMTS bearer level, for example due to header compression.

- $\quad$ Delivery order. This indicates whether the UMTS bearer shall provide in-sequence SDU (Service Data Unit) delivery or not and specifies if out-of-sequence SDUs are acceptable or not.

- $\quad$ Maximum SDU size used for admission control and policing. This corresponds to the maximum packet size that can be delivered at the top of the radio interface.

SDU format information. This is the list of possible exact sizes of SDUs.

SDU error ratio. This indicates the fraction of SDUs lost or detected as erroneous. This attribute is used to configure the protocols, algorithms and error detection schemes, primarily within UTRAN.

Residual bit error ratio. This indicates the undetected bit error ratio in the delivered SDUs. It is used to configure radio interface protocols, algorithms and error detection coding.

Delivery of erroneous SDUs. This indicates whether SDUs detected as erroneous will be delivered or discarded.

\section{Cellular Mobility Prioritization Schemes}

Different ideas and approaches are proposed to reduce the handover dropping probability. One approach is to reduce the handover failure rate is to prioritize handover call over new calls. Handover prioritization schemes have a significant impact on the call dropping probability and call blocking probability. Such scheme permits high utilization of bandwidth while guaranteeing the quality of service of handover calls.

The guard channel scheme was introduced in 80s for mobile cellular systems. However the guard channel scheme are still used in telecommunications with the name of Cut-off Priority Schemes. GC scheme improving the probability of successful handover by simply reserving a number of channels exclusively for handover in each cell. The GC scheme is feasible because new calls are less sensitive to delay than the handover calls.

According to GC Therefore according to the cell occupancy by Markov chain it is straight forward to derive the steady state probability $\mathrm{P} n$ that $\mathrm{n}$ channels are busy.

The call admission control scheme refers to the task of deciding whether new call requests are admitted into the network or not. In the CAC the arrival of new call are estimated continuously and if they are higher than the predefined threshold level then some calls are restricted (blocked) irrespective of whether a channel is available or not to decrease the probability of handover calls. In the CAC both the new and handover calls have to access to all channels. If a new call that is generated in cell cannot find an idle channel the call is discarded immediately.

In order to improve the mobility call prioritization scheme it is advisable to equalize the traffic load over the cells. Traffic reason and directed retry handover make use of this principal. First the new call to be served and if the receiver is able hear a neighbouring cell and are not considered in this situation. Traffic reason handover International Journal of Advance Research in Science and Engineering can be used to transfer traffic from one cell to another neighbouring when they are closed to the congestion. The traffic reason handover idea is based on the neighbouring cell having an overlapping service area. The overlapping service area arises naturally in GSM cellular system especially in small-cell high capacity micro cellular configurations. The small-cells are capture by subdividing a congested cell each with his own base station. The call arising in the common area (overlapping) of cells have access to channels more than one base station. By appropriate control strategy a cell may select the base station to establish a connation and contribute to efficient spectrum management. 


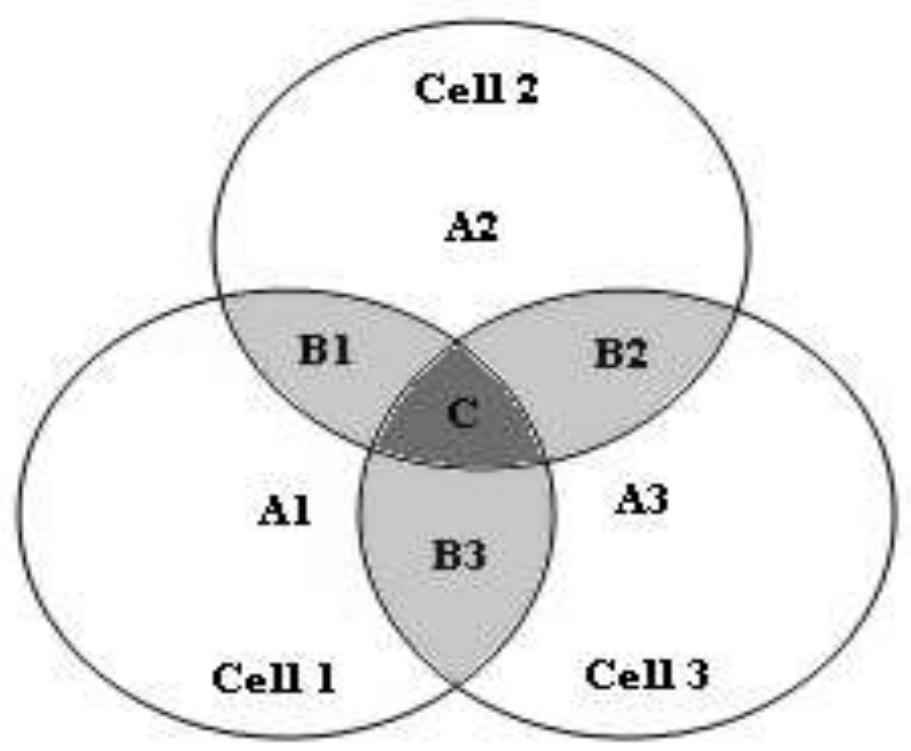

Fig 3: Areas A, B and C of three Cells

\section{QoS and QoE}

This section defines and explains the differences between QoS and QoE. This will help us to understand the requirements of the operator and of the end-user:

- QoS is defined as the ability of the network to provide a service at an assured service level. QoS encompasses all functions, mechanisms and procedures in the cellular network and terminal that ensure the provision of the negotiated service quality between the user equipment (UE) and the core network (CN).

- QoE is how a user perceives the usability of a service when in use - how satisfied he or she is with a service in terms of, for example, usability, accessibility, retainability and integrity of the service. Service integrity concerns throughput, delay, delay variation (or jitter) and data loss during user data transmission; service accessibility relates to unavailability, security (authentication, authorization and accounting), activation, access, coverage, blocking, and setup time of the related bearer service; service retainability, in general, characterizes connection losses.

QoS provisioning is a process that deploys QoS in networks and mobile terminals. The process translates planning results into mechanisms and parameters understandable by network elements and mobile terminals and it further configures them on equipment or devices.

QoS provisioning can be classified in three categories: radio, core and transport QoS provisioning that configures the QoS mechanisms inside the network; service QoS provisioning that maps services into QoS profiles and terminal QoS provisioning that provides service application specific QoS information to terminals.

With the growth of mobile services, it has become very important for an operator to measure the QoS and QoE of its network accurately and improve it further in the most effective and cost-efficient way to achieve customer loyalty and maintain competitive edge.

Cellular network optimization can be seen as a process to improve the overall network quality as experienced by the mobile subscribers and to ensure that the network resources are efficiently utilized. This includes performance measurements, analysis of measurement results and updates of the network configuration and parameters. The optimization process can be initiated because of several reasons, the most typical are:

- New technologies, elements or features of particular network elements are taken into use.

- $\quad$ External edge conditions have changed.

- Detection of decreased QoS performance in a particular network area.

\section{Roaming}

Roaming is derived from Real-time Optimally Adapting Mesh (ROAM). 
Roaming refers to a wireless network service extension in an area that differs from the registered home network location. Roaming enable a mobile device to access the internet and other mobile services when out of its normal coverage are. It also givers a mobile device the ability to move from one access point to another.

Roaming services are usually provided by cellular service providers as well as internet service providers (ISP) via a cooperative agreement. Traditional cellular roaming service are provided by both the global system for mobile communication (GSM) and Code Division Multiple Access (CDMA) operators. Services are either free or billed according to local area rates. Wireless telecommunication roaming series are usually included in mobile/cell phone subscriber services packages for use outside local networking zone. GSM/WLAN roaming services can be supplied in two different seniors. One in SIM - based roaming and the second in user-name/password base roaming.

Wireless local area network (WLAN) roaming servicers are segmented as follow.

Internal roaming: implemented when a mobile station is transferee with a strong signal between access points, preventing network blockage or interruption from weak signals.

External roaming: implemented when a mobile station shifts to a wireless land or other foreign wireless internet service provider (WISP) to a access services. WISP allows users to maintain an internet connection while mixing within a local coverage area.

\section{Paging}

The paging system was invented by Al Gross as an adaptation of his two - way radio, the walkie talkie. After some early market resistance from doctors who were afraid that the system would upset their patients and disturb their golf games, Gross sold the first system in 1950 to New York' s Jewish Hospital. That first system provided a means by which a centralized antenna could broadcast alerts to small, inexpensive pagers, or beepers. A page simply transmitted an identification number, which was recognized only by the pager being addressed. If that pager were in range, it beeped, hence the term beeper. Response to the page was in the form of a telephone call to the paging company to retrieve a message.

The downstream data to the pager originate in several ways. The most common approach involves the paging party dialing a telephone number which often is toll free. That number either may be dedicated to that one pager or may be one of many associated with the service provider.

Pagers can be classified as tone only, numeric, alphanumeric, and voice enabled. Tone - only pagers cause the device to emit an audible tone and/or to vibrate or blink so it is not disruptive. Numeric pagers permit the receipt of numbers only on a display. The vast majority of contemporary pagers are alphanumeric, capable of receiving and displaying both alphabetic and numeric characters. Contemporary pagers contain enough memory to support as many as 30,000 characters. But relatively few pagers currently can support the storage of voice messages, which are extremely memory intensive. This voice capability is accomplished through the downloading of compressed voice mail from a centralized voice processor to the pager over a packet network. Location

management protocols

For next-generation heterogeneous wireless networks, the inter-working and inter-operating function is suggested to accommodate roaming between dissimilar networks. For existing practical systems, several solutions are proposed for some specific pairs of interworking systems. In these schemes, the inter-operating function is implemented in either some additional inter-working unit with the help of dual-mode handsets.

In a location management mechanism has been proposed for heterogeneous network environment. It involves a mechanism for inter-system location updates and paging. Inter-system location update is implemented by using the concept of a boundary location area (BLA) existing at the boundary between two systems and off management is performed.

\section{Mobility Management Protocols}

A heterogeneous handover process in mobility management occurs when an MN either moves between networks with different access technologies, or between different domains. As the diversity of available networks increases, it is important that mobility technologies become agnostic to link layer technologies, and can operate in an optimized and secure fashion without incurring unreasonable delay and complexity. Supporting handovers across heterogeneous access networks, such as IEEE 802.11 (Wi-Fi), global system for mobile communications (GSM), code-division multiple access (CDMA), and worldwide interoperability for microwave access (WiMAX) is a challenge, as each has different quality of service (QoS), security, and bandwidth characteristics. Similarly, movement between different administrative domains poses a challenge since MNs need to perform access authentication and authorization in the new domain. Thus, it is desirable to devise a mobility optimization technique that can reduce these delays and is not tightly coupled to a specific mobility protocol. In this section, we describe different types of handovers and investigate the components that contribute to a handover delay. Some inter-technology and media-independent handover frameworks are then described. 


\section{Mobile services}

Sophisticated $4 \mathrm{G}$ service discovery mechanisms will combine the location/situation information and context-awareness in order to deliver users' services in a best possible manner. Additionally, future mobile services will require more complex personal and session mobility management to provision personalized services through different personalized operating environments to a single user terminal address. Whether SIP should be the core $4 \mathrm{G}$ protocol, and whether the service delivering framework be the network layer-based or application layer-based is still an open question Pandya et al (2010).

\section{Data Collection and Analysis}

In this study, various call quality parameters were evaluated for two cellular mobile operators, i.e operators $\mathrm{P}$, operator Q, with existing infrastructure within Semi-Urban Township Location in Nigeria. Two parameters were evaluated. These parameters are call setup time and performance statistics (QoS indicator). In this study, data collection was obtained from the network management centre (NMC) and all inclusive in mobile switching centres.

Data were obtained for the two cellular services operators, P and Q. Reports were initially collected and collated on hourly basis for which an average hourly figure was computed for a day. This progressively translated to an average of seven days taken for a week. Finally data were then computed on a weekly basis for a period of 24 weeks.

The following table shows the data obtained from each of the parameters.

Table 1. Call set up time

\section{S/N NETWORKS OPERATOR}

1. $\mathrm{P}$

2. $\mathrm{P}$

3. $\mathrm{Q}$

4. $\mathrm{Q}$
NETWORKS OPERATOR

$\mathrm{P}$

Q

Q

P
CALL SETUP TIME (h, $\mathbf{m}, \mathbf{s}, \mathbf{n s})$

00.00.07.20

00.00.10.02

00.00.08.07

00.00.08.22

Table 2: Performance statistics (QoS indicator)

$\begin{array}{llll}\text { S/N } & \text { QoS INDICATOR } & \mathbf{P}(\boldsymbol{\%}) & \mathbf{Q}(\%) \\ \text { 1. } & \text { TCH congestion (without handover) rate } & 4.15 & 0.87 \\ \text { 2. } & \text { TCH congestion (without handover) rate } & 20.00 & 5.10 \\ \text { 3. } & \text { Call set-up success rate } & 81.27 & 94.52 \\ \text { 4. } & \text { Drop call rate } & 1.41 & 1.62 \\ \text { 5. } & \text { SDDH congestion rate } & 4.15 & 2.34\end{array}$

Results

\section{Call Set Up Time}

From table 9 we observed that it takes $\mathrm{P}$ to $\mathrm{P}$ the shortest call set up time, this is followed by Q to P; Q to Q and finally it takes P to $\mathrm{Q}$ relatively the longest call set up time.

Call set up time was observed to be enhanced due to less congestion in the radio interface, effective radio resources allocation and access network transmission availability. 


\section{Performance Statistics (QoS Indicator)}

It is observed from table 2 that the channel congestion rate without handover management is higher respectively for P and Q. But with effective handover management the channel congestion becomes lower. Also the causes of success rate for P and Q are relatively high and efficient. Finally, while $\mathrm{P}$ recorded lower drop call rate, $\mathrm{P}$ however is higher than $\mathrm{Q}$ channel congestion rate.

Quality of service played significant roles in attaining network interconnectivity and operation reliability.

\section{Discussion}

In today's competitive telecommunication environment, mobility management is the most important feature of a wireless cellular communication system.

In cellular communication today, emphasis has shifted to delivery of exceptional services while maintaining low cost and operation reliability. The telecommunication service providers are saddled with the challenge to deliver high quality service, innovative at a time of rapid change and cost pressure. Improved network availability, reliability and effective handover management can play significant roles in attaining the goal of sustained interconnectivity and maintainability.

Mobility management is a resource for the information needed to manage the networks in a proactive way so the service problem can be addressed before it affects the customer. Much of raw data about various network elements is not useful until it is translated into real information as observed between this study and related earlier studies.

\section{Conclusion}

The mobility management within any cellular system is of great importance. It is a critical process and if performed incorrectly handover can result in the loss of the call.

In this study a great deal of emphasis on mobility in GSM network was given .Very important too is the fact that handover in GSM network are very important to maintain the quality of a call. Mobility prioritization schemes were also analyzed in order to enhance the quality of service (QoS) of GSM wireless network. Extensive survey and analysis of the handover prioritization schemes that is guard channels, call admission control and handover queuing has been provided. This research work contributed the following to telecommunication body of knowledge and the larger society among others:

Presentation of an cellular mobility framework to enhance efficient service delivery in wireless network and exploration of some of the most time proven cellular communication protocols to meet the competitive demands of data rates and spectral capacity.

\section{Recommendations}

The following are hereby recommended for greater achievement in further research;

(1) To predict the upcoming traffic evolution and network expansions as per increasing number of mobile users.

(2) To benchmark network with another competitor's network to attract more users at the cost of better quality on a national and global platform.

\section{REFERENCES}

Akyildiz, I.F.; Altunbasak, Y. and Sivakumar, R. (2012). AdaptNet: an adaptive protocol suite for the next generation wireless internet. IEEE Communications Journal 42: 128 - 136.

Buddhikot, M.; Chandranmenon, G.; Han, S. and Lee, Y.W. (2011). Integration of 802.11 and Third-Generation Wireless Data Networks. IEEE INFOCOM Journal 1: 503 - 512.

Campbell, A.T.; Gomez, J. and Kim, S. (2011). Design, implementation and evaluation of cellular IP. IEEE Personal Communications Journal 7: $42-49$.

Dutta, A.; Famolari, D.; Das, S. and Ohba, Y. (2012). Media-independent pre-authentication supporting secure inter domain handover utilization. IEEE Wireless Communication Journal 15: 55 - 64 .

Eastwood, L.; Migaldi, S.; Xie, Q. and Gupta, V. (2010). Mobility using IEEE 802.21 in a heterogeneous IEEE 802.16/802.11based IMT-advanced (4G) network. IEEE Wireless Communication Journal 15: 26 - 34.

Ekici, E. (2012). Optimal Two-Tier Cellular Network Design. IEEE Wireless Communication Journal 10: 24 - 32. 
Ghaderi, M. and Boutaba (2010). Call Admission Control in Mobile Cellular Networks: A Comprehensive Survey. Wireless Communications and Mobile Computing Journal 6: 69 - 93.

Hsieh, R.; Zhou, Z.G. and Seneviratne, A. (2011). A seamless handoff architecture for Mobile IP. IEEE INFOCOM Journal 3: 174 -184 .

Kim, K.; Kim, C. and Kim, T. (2012). A seamless handover mechanism for IEEE 802.16e broadband access. International Journal on Computational Science 3515: 527534.

Misra, A.; Das, S. and McAuley, A. (2010). IDMP - based fast handoffs and paging in IP-based 4G mobile networks. IEEE Communication Journal 4: 138 - 145.

Misra, A.; Das, S.; Dutta, A.; McAuley, A. and Das, S.K. (2002). IDMP-based fast handoffs and paging in IP-based 4G mobile networks. IEEE Communications Magazine 4: 138-145.

Mohanty, S. (2011). A new architecture for 3G and WLAN integration and inter-system handover management. Wireless Networks Journal 12: $733-745$.

Pandya, R.; Grillo, D. and Lycksell, E. (2010). IMT - 20000 standards: networks aspects. IEEE Personal Communication Journal 4: $20-29$.

Salsano, S.; Polidoro, A.; Mingardi, C.; Niccolini, S. and Veltri, L. (2008). SIP-based mobility management in next-generation networks. IEEE Wireless Communications 15: 92-99.

Soliman, H.; Castelluccia, C.; ElMalki, K. and Bellier, L. (2008). Hierarchical Mobile IPv6 mobility management (HMIPv6). IEEE Wireless Communications Journals 15: 81 - 84

Yokota, H.; Idoue, A.; Hasegawa, T. and Kato, T. (2002). Link layer assisted Mobile IP fast handoff method over wireless LAN networks. Proceedings of ACM MOBICOM 11: 131-139.

Zhang, Y. (2008). Vertical handoff between 802.11 and 802.16 wireless access networks. IEEE Communications Journal 15: 91 94.

Zhang, Q.; Guo, C.; Guo, Z. and Zhu, W. (2003). Efficient mobility management for vertical handoff between WWAN and WLAN. IEEE Communications Journal 11: 102-108. 